\title{
Video Article \\ A New Screening Method for the Directed Evolution of Thermostable Bacteriolytic Enzymes
}

\author{
Ryan D. Heselpoth ${ }^{1}$, Daniel C. Nelson ${ }^{1}$ \\ ${ }^{1}$ Institute for Bioscience and Biotechnology Research, University of Maryland \\ Correspondence to: Daniel C. Nelson at nelsond@umd.edu \\ URL: https://www.jove.com/video/4216 \\ DOI: doi:10.3791/4216
}

Keywords: Immunology, Issue 69, Molecular Biology, Genetics, Microbiology, directed evolution, thermal behavior, thermostability, endolysin, enzybiotic, bacteriolytic, antimicrobial, therapeutic, PlyC

Date Published: 11/7/2012

Citation: Heselpoth, R.D., Nelson, D.C. A New Screening Method for the Directed Evolution of Thermostable Bacteriolytic Enzymes. J. Vis. Exp. (69), e4216, doi:10.3791/4216 (2012).

\section{Abstract}

Directed evolution is defined as a method to harness natural selection in order to engineer proteins to acquire particular properties that are not associated with the protein in nature. Literature has provided numerous examples regarding the implementation of directed evolution to successfully alter molecular specificity and catalysis ${ }^{1}$. The primary advantage of utilizing directed evolution instead of more rational-based approaches for molecular engineering relates to the volume and diversity of variants that can be screened ${ }^{2}$. One possible application of directed evolution involves improving structural stability of bacteriolytic enzymes, such as endolysins. Bacteriophage encode and express endolysins to hydrolyze a critical covalent bond in the peptidoglycan (i.e. cell wall) of bacteria, resulting in host cell lysis and liberation of progeny virions. Notably, these enzymes possess the ability to extrinsically induce lysis to susceptible bacteria in the absence of phage and furthermore have been validated both in vitro and in vivo for their therapeutic potential ${ }^{3-5}$. The subject of our directed evolution study involves the PlyC endolysin, which is composed of PlyCA and PlyCB subunits ${ }^{6}$. When purified and added extrinsically, the PlyC holoenzyme lyses group A streptococci (GAS) as well as other streptococcal groups in a matter of seconds and furthermore has been validated in vivo against GAS ${ }^{7}$. Significantly, monitoring residual enzyme kinetics after elevated temperature incubation provides distinct evidence that PlyC loses lytic activity abruptly at 45 ${ }^{\circ} \mathrm{C}$, suggesting a short therapeutic shelf life, which may limit additional development of this enzyme. Further studies reveal the lack of thermal stability is only observed for the PlyCA subunit, whereas the PlyCB subunit is stable up to $\sim 90{ }^{\circ} \mathrm{C}$ (unpublished observation). In addition to PlyC, there are several examples in literature that describe the thermolabile nature of endolysins. For example, the Staphylococcus aureus endolysin LysK and Streptococcus pneumoniae endolysins $\mathrm{Cpl}-1$ and Pal lose activity spontaneously at $42{ }^{\circ} \mathrm{C}, 43.5^{\circ} \mathrm{C}$ and $50.2^{\circ} \mathrm{C}$, respectively $y^{8-10}$. According to the Arrhenius equation, which relates the rate of a chemical reaction to the temperature present in the particular system, an increase in thermostability will correlate with an increase in shelf life expectancy ${ }^{11}$. Toward this end, directed evolution has been shown to be a useful tool for altering the thermal activity of various molecules in nature, but never has this particular technology been exploited successfully for the study of bacteriolytic enzymes. Likewise, successful accounts of progressing the structural stability of this particular class of antimicrobials altogether are nonexistent. In this video, we employ a novel methodology that uses an error-prone DNA polymerase followed by an optimized screening process using a 96 well microtiter plate format to identify mutations to the PlyCA subunit of the PlyC streptococcal endolysin that correlate to an increase in enzyme kinetic stability (Figure 1). Results after just one round of random mutagenesis suggest the methodology is generating PlyC variants that retain more than twice the residual activity when compared to wild-type (WT) PlyC after elevated temperature treatment.

\section{Video Link}

The video component of this article can be found at https://www.jove.com/video/4216/

\section{Protocol}

\section{Determining Optimal Heating Conditions}

First, one must experimentally determine the optimal incubation temperature and time to use for the heating step in the assay. For our PlyC model, it is important to note that $E$. coli co-transformed with plyCA and plyCB genes on separate expression plasmids has been shown to form a fully functional PlyC holoenzyme ${ }^{6}$. The 96 well microtiter plate preparation as well as cell growth conditions and the subsequent replica plating technique were adapted from examples provided in the literature ${ }^{12-16}$. An incubation time of 30 min will be suitable for this assay as results from previous thermal inactivation experiments dictate a rapid loss in activity during short-term incubation in environments exceeding physiological temperature (unpublished observation). The optimal incubation temperature for PlyC was elucidated by the following steps:

1. Transform the expression construct pBAD24:plyCA (Amp $\left.{ }^{r}\right)$ into the competent $E$. coli strain DH5a pBAD33:plyCB (Cm $\left.{ }^{r}\right)$.

2. Plate the transformants on a Luria-Bertani (LB) agar plate supplemented with ampicillin $(100 \mu \mathrm{g} / \mathrm{ml})$ and chloramphenicol $(35 \mu \mathrm{g} / \mathrm{ml})$. Incubate the plates overnight at $37^{\circ} \mathrm{C}$. 
3. With a sterile toothpick, inoculate an individual colony into each row of wells (Figure 2a) in a sterile, clear, flat-bottomed 96 well, lidded microtiter plate that contains $200 \mu \mathrm{l}$ of LB supplemented with ampicillin and chloramphenicol.

4. Securely place the 96 well microtiter plate on a $37^{\circ} \mathrm{C}$ shaking incubator and grow the bacteria overnight at $300 \mathrm{rpm}$.

5. Retrieve the 96 well microtiter plate from the $37^{\circ} \mathrm{C}$ shaking incubator and replica plate to a new 96 well microtiter plate as follows:

a. Add $180 \mu \mathrm{l}$ of LB supplemented with ampicillin and chloramphenicol to each well of the replica plate.

b. Transfer $20 \mu \mathrm{l}$ of bacteria from the original plate to the corresponding wells in the replica plate. This should result in an initial $\mathrm{OD}_{600}$ of $\sim 0.5$.

6. Securely place the replica plate on the $37{ }^{\circ} \mathrm{C}$ shaking incubator and incubate the plate for $1 \mathrm{hr}$ at $300 \mathrm{rpm}$, then add the inductant. In the case of the pBAD expression systems, the inductant is $0.25 \%$ arabinose. Other expression systems may require different inductants. Place the plate back on the $37^{\circ} \mathrm{C}$ shaking incubator and shake at $300 \mathrm{rpm}$ for an additional $4 \mathrm{hr}$ to allow for protein expression. Expression levels typically range between $10-20 \mu \mathrm{g}$ of PlyC.

7. Once protein expression has concluded, retrieve the replica plate and pellet the bacteria by placing the 96 well microtiter plate in a refrigerated centrifuge that contains a swinging-bucket rotor that fits 96 well microtiter plates. Centrifuge at $3,000 \mathrm{rpm}$ for $10 \mathrm{~min}$ at room temperature.

8. Remove the media supernatant by slowly inverting the microtiter plate and gently decanting the media.

9. Lyse the cells by adding $50 \mu \mathrm{l}$ of B-PER II Bacterial Protein Extraction Reagent to each well. Incubate the plate at room temperature for 20 $\min$.

10. Increase the volume in each well to $120 \mu \mathrm{l}$ by adding $70 \mu \mathrm{l}$ of phosphate buffered saline (PBS) pH 7.2.

11. Pellet the insoluble cellular debris by spinning the plate at $3,000 \mathrm{rpm}$ for $10 \mathrm{~min}$ at $4{ }^{\circ} \mathrm{C}$ in the refrigerated centrifuge.

12. Transfer $110 \mu \mathrm{l}$ of the soluble crude lysate to the corresponding wells of a 96 well thermocycler plate.

13. Using $30 \mathrm{~min}$ as the predetermined incubation time, expose the soluble lysates currently residing in the 96 well thermocycler plate to a broad gradient temperature range spanning $15-20^{\circ} \mathrm{C}$. Note, the goal is to determine at what temperature a particular enzyme loses $>95 \%$ catalytic activity.

14. After incubation, incubate the 96 well thermocycler plate at $4{ }^{\circ} \mathrm{C}$ for $5 \mathrm{~min}$ and transfer $100 \mu \mathrm{l}$ of the soluble lysates from the 96 well thermocycler plate to their corresponding well location in the final 96 well microtiter plate.

15. With a 12-channel multipipettor, add $100 \mu$ of GAS strain D471 to each well. Note, D471 cells are originally lyophilized from overnight cultures and resuspended with PBS pH 7.2 to obtain an $\mathrm{OD}_{600}$ of $\sim 2.0$.

16. Immediately place the 96 well plate in the microplate spectrophotometer and monitor the enzyme kinetics by measuring the $\mathrm{OD}_{600}$ every 15 $\mathrm{sec}$ for $20 \mathrm{~min}$. The lowest temperature incubation that corresponded to wells that lacked a change in optical density $(\Delta O D \leq 0.1)$ is defined as the non-permissive temperature.

After a broad temperature screen is performed to identify the non-permissive temperature, the above steps can be repeated over a more narrow range of temperatures $\left(5^{\circ}-10^{\circ} \mathrm{C}\right)$ to elucidate the precise non-permissive temperature for the enzyme of interest. Note, the non-permissive temperature identified in this assay may be different than the melting temperature elucidated by other means due to differences in volume, concentration, etc.

\section{Generating the Mutant Library}

The creation of the mutant library to be screened involves randomly incorporating mutations by an error-prone DNA polymerase with minimal nucleotide bias in the plyCA gene using the GeneMorph II Random Mutagenesis Kit as follows:

1. Design nucleotide primers with similar melting temperatures $\left(T_{m}\right)$ between $55-72{ }^{\circ} \mathrm{C}$ with the restriction sites of choice at the $5^{\prime}$ and $3^{\prime}$ ends of the plyCA gene.

2. Since the desired mutation rate is $2-3$ nucleotides per plyCA $(1.4 \mathrm{~kb})$, use the PCR reaction component concentrations as well as the thermocycler conditions recommended by the manufacturer for low mutation frequencies (0-4.5 per kb).

3. Clone the mutagenized plyCA genes into the expression vector pBAD24.

4. Transform the constructs into a DH5a pBAD33:plyCB background and plate transformants on LB agar plates supplemented with ampicillin and chloramphenicol.

5. Additionally, transform and plate $\mathrm{DH} 5 \alpha$ pBAD33:plyCB with the expression vector pBAD24 containing the WT plyCA gene. Colonies from this plate will serve as the controls during screening.

\section{96 Well Microtiter Plate Preparation and Cell Growth Conditions}

1. Fill each well of a 96 well microtiter plate with $200 \mu$ l of LB supplemented with ampicillin and chloramphenicol.

2. Following the microtiter plate schematic (Figure $\mathbf{2 b}$ ), carefully select an individual colony from the overnight agar plates with a sterilized toothpick and inoculate the bacteria in the designated well of the 96 well microtiter plate. It is essential to ensure only one colony per well is inoculated.

3. Shake the securely anchored 96 well microtiter plate at $300 \mathrm{rpm}$ overnight at $37^{\circ} \mathrm{C}$.

\section{Replica Plating, Protein Expression Induction and Lysate Preparation}

1. Follow steps 1.5-1.11 from the section titled "Determining the optimal heating conditions" with one modification. Store the original plate at 4 ${ }^{\circ} \mathrm{C}$ after the replica plating step has concluded.

2. After step 1.11, transfer $110 \mu \mathrm{l}$ of the soluble crude lysate to the corresponding wells of a 96 well thermocycler plate except for the positive control wells A1-D1. With regard to the positive control lysates (i.e. lysates that are not heated), transfer $100 \mu$ l of the lysates to the corresponding wells in a new 96 well microtiter plate that will serve as the final assay plate for the experiment and store on ice. 


\section{Soluble Lysate Heat Treatment}

1. Heat the negative control and mutant soluble lysates currently confined in the 96 well thermocycler plate in the thermocycler for 30 min at the optimized non-permissive incubation temperature determined in step 1.

2. After non-permissive temperature incubation, incubate the 96 well thermocycler plate at $4{ }^{\circ} \mathrm{C}$ for $5 \mathrm{~min}$.

3. Transfer $100 \mu \mathrm{l}$ of the soluble lysates from the 96 well thermocycler plate to their identical well locations in the final 96 well microtiter assay plate already containing the positive control soluble lysates.

4. With a 12-channel multipipettor, add $100 \mu$ of GAS strain D471 to each well. Immediately place the plate in the microplate spectrophotometer.

5. Monitor the enzyme kinetics by measuring the $\mathrm{OD}_{600}$ every $15 \mathrm{sec}$ for $20 \mathrm{~min}$.

a. A PlyC variant with progressed thermal behavior is defined as a construct that can decrease the original optical density by 50 percent in less than $900 \mathrm{sec}$ at the non-permissive temperature. Additionally, the positive controls (i.e. non-heated, WT PlyC) need to display WT catalytic activity $\left(t_{1 / 2} \leq 100 \mathrm{sec}\right)$ and the negative controls (i.e. heated, WT PlyC) should be devoid of activity.

6. Once a PlyC variant with progressed thermal behavior is identified, retrieve the original plate stored at $4{ }^{\circ} \mathrm{C}$ and inoculate bacteria from the individual well specific to the mutant of interest into fresh LB media supplemented with ampicillin and chloramphenicol. Grow the inoculum overnight at $37^{\circ} \mathrm{C}$.

7. Extract and purify plasmid DNA from the culture and then submit for sequencing in order to identify the nucleotide mutations that infer enhanced thermal behavior to PlyCA. Additionally, supplement a small aliquot from the overnight culture with $10 \%$ glycerol and store at -80 ${ }^{\circ} \mathrm{C}$ for further use.

\section{Representative Results}

At the conclusion of the first round of random mutagenesis, over 6,000 PlyC mutants were screened and a total of 35 mutants with potentially increased thermal behavior were identified, selected and sequenced. Genomic analysis, summarized in Table I, suggests that of the 35 candidates, 7 of the constructs contained WT PlyCA sequences at the level of translation, corresponding to false positives identified by the assay. Of the remaining 28 candidates, the mutation range was from 1 to 6 nucleotide mutations with an average mutation rate of 2.75 nucleotides per plyCA gene, which was in the 2-3 nucleotide mutation range we were targeting. At the translational level, this particular nucleotide mutation range and frequency yielded an amino acid mutation range of 1 to 5 amino acids, with an average mutation rate of 1.9 amino acid mutations per PlyCA polypeptide.

Of the 28 candidates with at least one amino acid mutation, four of these mutant constructs were randomly chosen for further characterization to validate that the extensive screening process of the directed evolution methodology was indeed functioning properly. The mutant PlyC enzymes were purified to $>95 \%$ homogeneity based on SDS-PAGE analysis as previously described ${ }^{6-7}$. Enzyme kinetics of WT PlyC and each of the four PlyC mutants were characterized at equal molar concentrations after incubating the purified enzymes at various elevated temperatures. Activity was monitored after the addition of D471 GAS by measuring the optical density at $600 \mathrm{~nm}$ every $15 \mathrm{sec}$ for 20 min. Activity was defined as the residual maximum velocity of the enzyme after heat incubation. Of the four candidates randomly selected for further characterization, mutant $29 \mathrm{C} 3$ showed the most enhanced thermal behavior. The thermal behavior of WT PlyC and 29C3 was investigated at different temperatures while incubating and assaying for activity in PBS pH 7.2 at $80 \mathrm{nM}$ and $40 \mathrm{nM}$ concentrations, respectively. The $45-50{ }^{\circ} \mathrm{C}$ incubation experiments (Figure 3) were performed in a thermocycler where the samples were incubated in a thin-walled 96 well thermocycler plate in a total volume of $120 \mu \mathrm{l}$. The $35^{\circ} \mathrm{C}, 40^{\circ} \mathrm{C}$ and $45^{\circ} \mathrm{C}$ incubation experiments (Figure 4 and 5) were performed in a heat block where the samples were incubated in a $1.5 \mathrm{ml}$ microcentrifuge tube in a total volume of $1.3 \mathrm{ml}$. All experiments were performed in triplicates.

WT PlyC and $29 \mathrm{C} 3$ showed no significant difference in kinetic stability at room temperature $\left(25^{\circ} \mathrm{C}\right)$ as depicted in the first set of bars in Figure 3. However, after a short-term incubation for $30 \mathrm{~min}$ from temperatures ranging from $45-50{ }^{\circ} \mathrm{C}$, the activity of $29 \mathrm{C} 3$ was substantially greater than the activity specific to the WT construct at each temperature point. For example, WT PlyC displayed a $44 \%$ loss in activity at $45.2{ }^{\circ} \mathrm{C}$ whereas $29 \mathrm{C} 3$ showed only a $2 \%$ loss in activity at the same temperature.

Long-term incubation studies comparing the residual activity of both WT PlyC and $29 \mathrm{C} 3$ were additionally performed at $35{ }^{\circ} \mathrm{C}$ and $40{ }^{\circ} \mathrm{C}$ involving the measurement of residual activity at 24 and $48 \mathrm{hr}$ time points. At $35^{\circ} \mathrm{C}, 29 \mathrm{C} 3$ displayed $41 \%$ and $176 \%$ higher activity than WT PlyC at 24 and $48 \mathrm{hr}$ incubation time points, respectively (Figure 4a). At $40{ }^{\circ} \mathrm{C}, 29 \mathrm{C} 3$ displayed $28 \%$ and $107 \%$ higher activity than WT PlyC at 24 and $48 \mathrm{hr}$ incubation time points, respectively (Figure $\mathbf{4 b}$ ).

The residual activity of WT PlyC and $29 \mathrm{C} 3$ were also monitored every $20 \mathrm{~min}$ for a total of $3 \mathrm{hr}$ at $45^{\circ} \mathrm{C}$. WT PlyC was only able to retain $21 \%$ activity after a $3 \mathrm{hr}$ incubation at this temperature whereas $29 \mathrm{C} 3$ was able to retain $46 \%$ activity. The half-life ( $\mathrm{t}_{1 / 2}$ ) for WT PlyC and $29 \mathrm{C} 3$ were 67 and $147 \mathrm{~min}$, respectively, suggesting that $29 \mathrm{C} 3$ has a 2.2 fold increase in kinetic stability at $45^{\circ} \mathrm{C}$ (Figure 5).

\begin{tabular}{|l|l|}
\hline Total Round 1 Candidates & 35 \\
\hline Candidates with WT PlyCA Sequence & 7 \\
\hline Candidates with $\geq 1$ Amino Acid Mutation & 28 \\
\hline - Average Nucleotide Mutation Rate (nt/plyCA) & 2.75 \\
\hline - Nucleotide Mutation Range (nt) & $1-6$ \\
\hline - Average Amino Acid Mutation Rate (AA/PlyCA) & 1.9 \\
\hline - Amino Acid Mutation Range (AA) & $1-5$ \\
\hline
\end{tabular}

Table 1. Candidate Pool Genomic Analysis. 


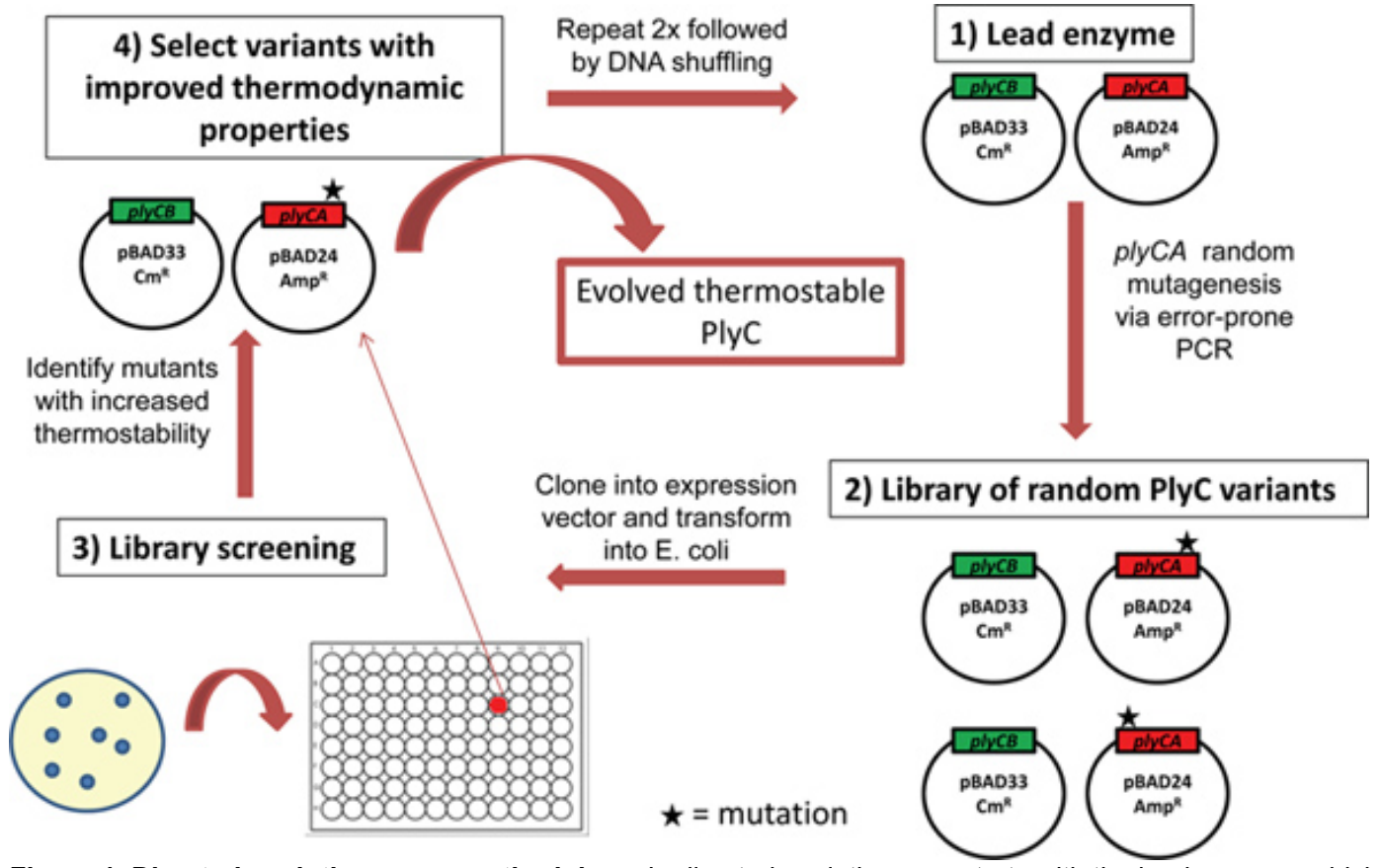

Figure 1. Directed evolution assay methodology. In directed evolution, one starts with the lead enzyme, which would be WT PlyC for the first round. A library of PlyC mutants containing random unbiased nucleotide mutations to the plyCA gene is then constructed by an error-prone DNA polymerase, cloned into the expression vector pBAD24 and transformed into the $E$. coli strain DH5a already containing pBAD33:plyCB. Individual transformants are inoculated into their own specific well of a 96 well microtiter plate. Through an extensive screening process, individual PlyC mutants that are catalytically active after incubation at a non-permissible temperature are classified as mutants with enhanced kinetic stability. Theconstruct displaying the most progressed thermal behavior will consequently becomes the lead enzyme for the next round of random mutagenesis. Overall, there are three complete rounds of random mutagenesis followed by DNA shuffling resulting in a bacteriolytic molecule with evolved thermal behavior. Click here to view larger figure. 
A.

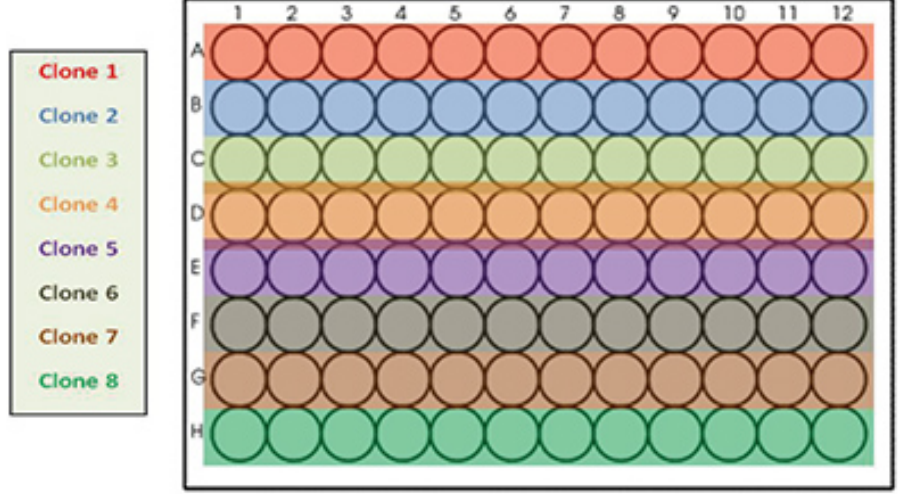

B.

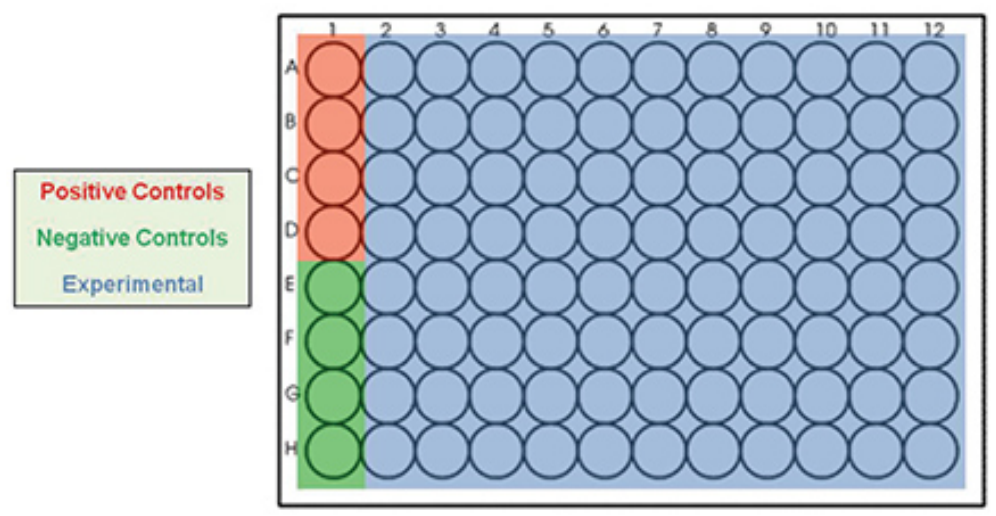

Figure 2. 96 well microtiter plate templates for directed evolution assay. The microtiter plate schematic during the determination of the optimal heating conditions (Figure 2a) consists of inoculating a single parental WT PlyC clone into each row of the microtiter plate. The microtiter plate schematic during mutant screening (Figure 2b) consists of inoculating each well in column 1 with a unique parental WT PlyC clone as well as inoculating each well in columns 2-12 with a distinct PlyC mutant clone. Wells A1-D1 are designated for the positive parental controls, which consist of WT PlyC constructs not exposed to non-permissible temperature incubation. Wells E1-H1 are designated for the negative parental controls, which consist of WT PlyC constructs that are exposed to non-permissible temperature incubation.

\section{Enzyme Residual Activity}

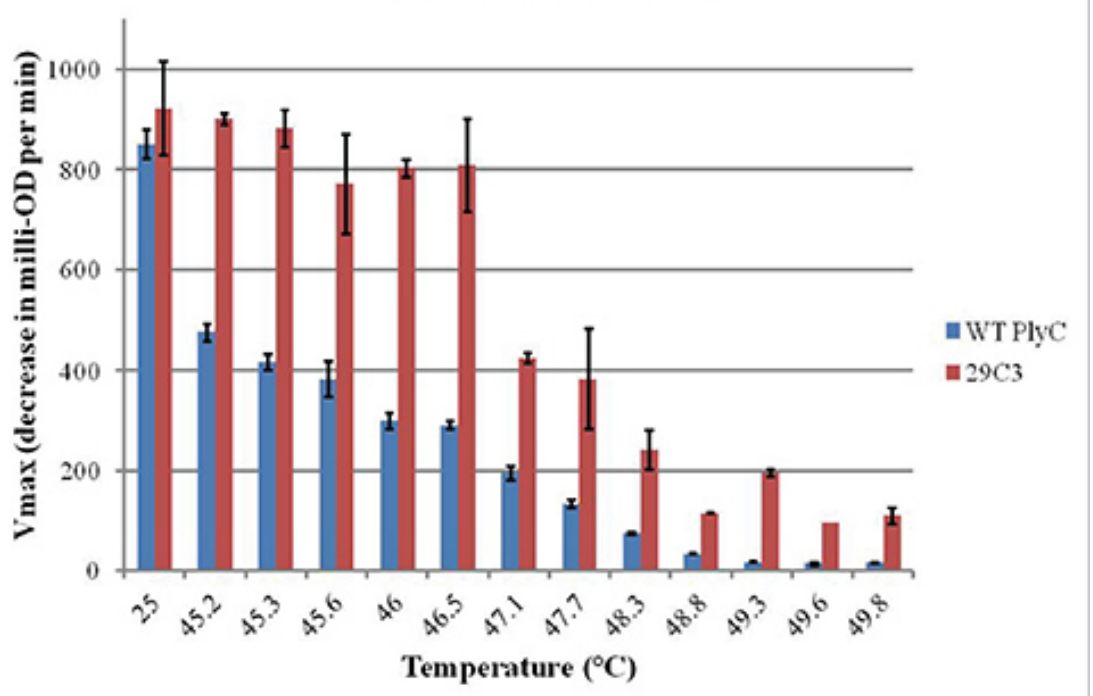

Figure 3. Residual activity kinetic analysis comparing WT PlyC and 29C3. Enzymes were purified to homogeneity and incubated for 30 $\mathrm{min}$ in a thermocycler at equal molar concentrations at either room temperature or at a temperature gradient ranging from $45-50^{\circ} \mathrm{C}$. Enzymatic activity correlates with the maximum velocity displayed after specific temperature incubation. With the exception of $25^{\circ} \mathrm{C}$ and $47.7^{\circ} \mathrm{C}$, the variation in activity between WT and $29 \mathrm{C} 3$ at each temperature correlated to a p-value $<0.05$. All data are reported as the mean \pm SEM of three independent experiments. 
A.

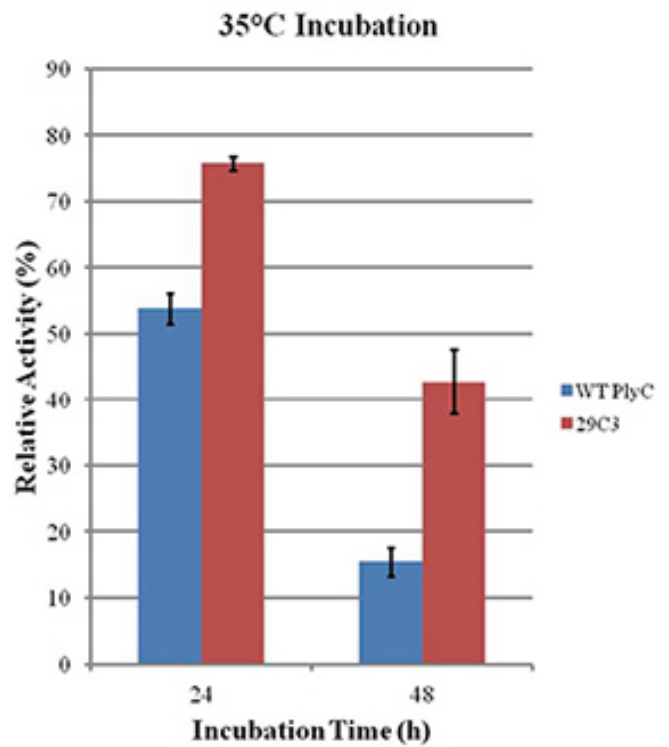

B.

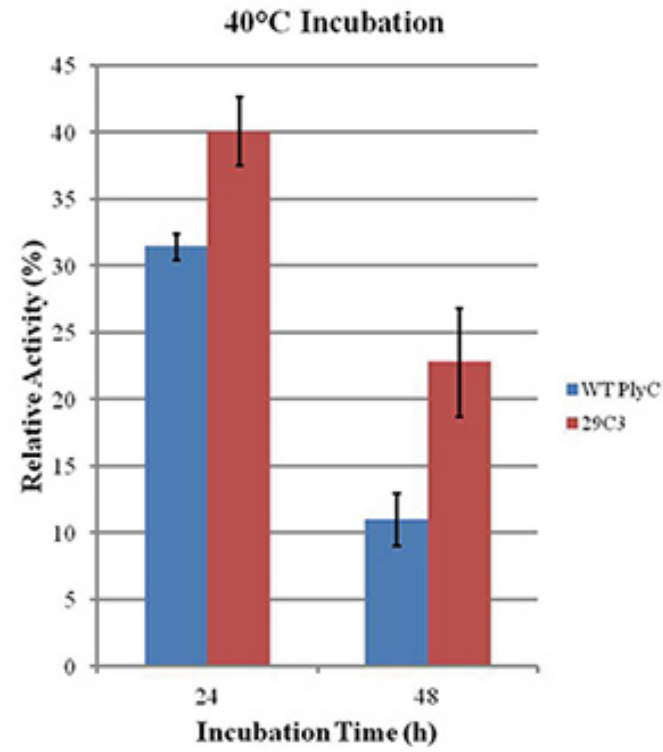

Figure 4. Residual activity kinetic analysis comparing WT PlyC to $29 \mathrm{C} 3$ at $35^{\circ} \mathrm{C}$ and $40{ }^{\circ} \mathrm{C}$. Equal molar concentrations of purified WT PlyC and $29 \mathrm{C} 3$ were incubated in a heat block at $35^{\circ} \mathrm{C}$ (Figure $4 \mathrm{a}$ ) or $40^{\circ} \mathrm{C}$ (Figure $4 \mathbf{b}$ ). The residual enzyme activity was measured at 24 and $48 \mathrm{hr}$ time points. The activity displayed by each construct was normalized to the maximum velocity displayed at time point zero. The variation in activity between WT and $29 \mathrm{C} 3$ at each temperature and time point correlated to a p-value $<0.05$. All data are reported as the mean \pm SEM of three independent experiments.

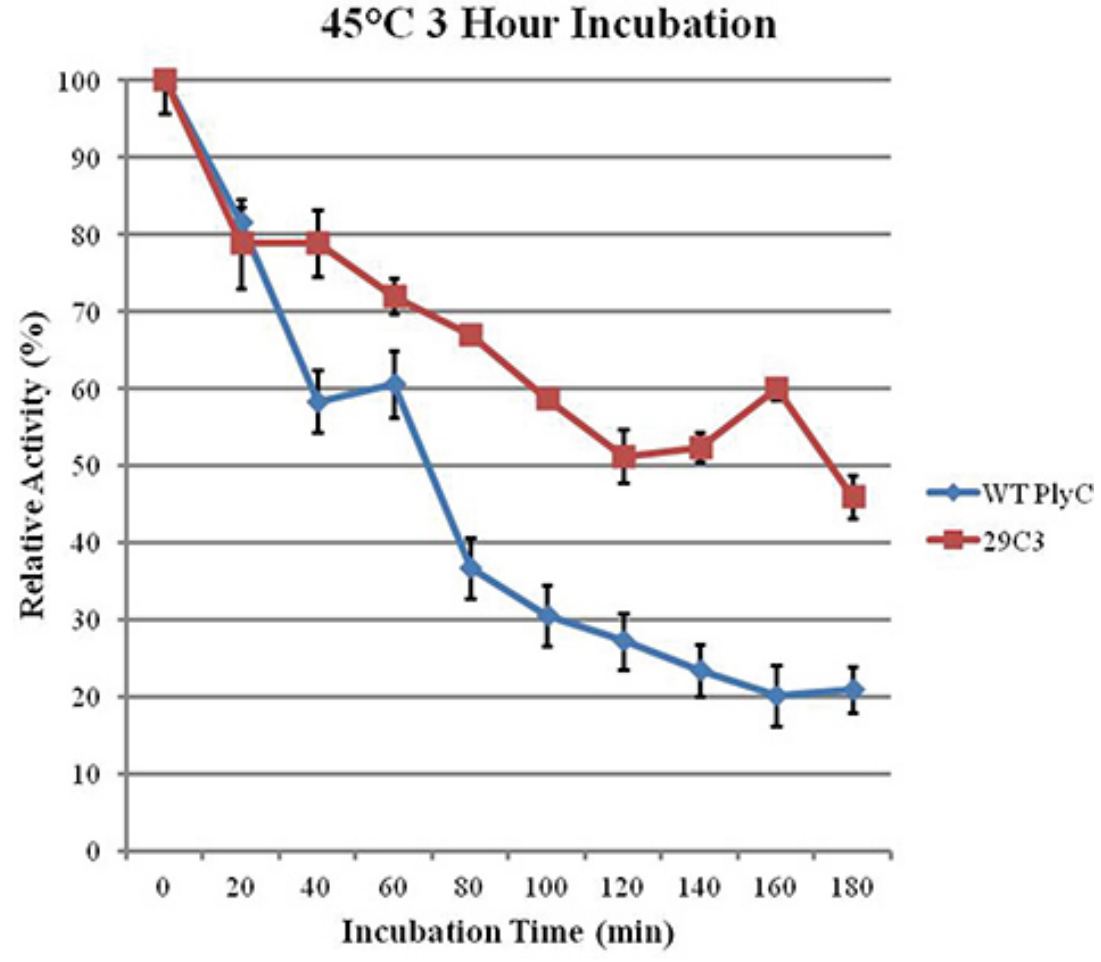

Figure 5. Residual activity kinetic analysis comparing WT PlyC to $29 \mathrm{C} 3$ at $45^{\circ} \mathrm{C}$. Equal molar concentrations of purified WT PlyC and $29 \mathrm{C} 3$ were incubated in a heat block at $45^{\circ} \mathrm{C}$ and the residual enzyme activity was measured every 20 min for 3 hr. The activity displayed by each construct was normalized to the maximum velocity displayed at time point zero. With the exception of the data points at 20 min, the variation in activity between WT and $29 \mathrm{C} 3$ at each time point correlated to a p-value $<0.05$. All data are reported as the mean \pm SEM of three independent experiments.

\section{Discussion}

This protocol, outlined in Figure 1, presents a 96 well microtiter plate methodology that allows one to utilize directed evolution to increase the thermostability of any bacteriolytic enzyme. Through the use of an error-prone DNA polymerase, one can introduce random mutations 
that increase the overall kinetic stability of the translated bacteriolytic molecule of interest, which is typically due to molecular reorganizations consisting of increasing electrostatic, disulphide bridge and hydrophobic interactions that generate improved molecular packing, enhanced modifications of surface charge networks or reinforcement of a higher oligomerization state ${ }^{17-18}$. After the introduction of nucleotide mutations, an extensive screening procedure was then used to identify mutations that are thermally beneficial. The representative results presented here were based on one round of random mutagenesis. In reality, it has been suggested that at least three successive rounds of random mutagenesis, each using the most robust mutant as the lead enzyme, followed by DNA shuffling is appropriate for these types of directed evolution thermal behavior studies ${ }^{2}$.

It is important to understand that while this protocol identifies mutations that augment kinetic stability, these variants do not necessarily have increased thermostability. A molecule is considered thermostable when it has the ability to retain its structure at a high temperature. However, in the assay presented, the residual activity of the mutant lysates are not assayed at the same temperature that they were initially incubated at during the heat treatment step and thus one may be selecting for mutations that promote refolding rather than enhanced thermostability ${ }^{19}$. While we are extrapolating thermal behavior as an indication of thermal stability, true thermal stability must be measured empirically by biophysical methods such as circular dichroism (CD), differential scanning calorimetry (DSC) and differential scanning fluorimetry (DSF). Preliminary data from CD and DSF experiments suggest that several candidates identified from the methodology presented do indeed display progressed thermostability (data not shown).

Although the methodology presented here is specific to implementing increased thermal behavior to PlyC, this same methodology can additionally be employed to enhance thermal activity to other bacteriolytic enzymes with some minor alterations to variables such as buffers, mutation rates, heating conditions and expression systems. One critical variable associated with this assay relates to the nucleotide mutation rate of the error-prone DNA polymerase. We chose to use the error-prone Mutazyme II DNA polymerase in our directed evolution assay due to experimental evidence suggesting this particular enzyme displays the least amount of bias with regards to which nucleotides are randomly incorporated into the gene of interest when compared to other random mutagenesis techniques such as use of hydroxylamine, mutator $E$. coli strains and other error-prone DNA polyermases ${ }^{20}$. In general, lower mutation rates tend to be more desirable for two reasons. First, engineering thermostability to proteins typically consists of relatively few amino acid substitutions. High mutation rates can cause dramatic structural alterations to particular molecules that can conclusively result in structural misfolding and functional discrepancies. For example, the incorporation of glycine and proline residues can disrupt alpha helical secondary structures ${ }^{21}$. Second, high nucleotide mutation rates increase the chances of incorporating premature stop codons, resulting in truncated molecules that are biologically inactive. In general, lower mutation rates are preferred because lower error rates result in the accumulation of adaptive mutations while higher mutation rates generate neutral or deleterious mutations ${ }^{22}$.

For each round of random mutagenesis, another critical variable that one must optimize involves the incubation temperature used during the screening process. Selection of the experimental non-permissive temperature is relatively challenging. We initially used an incubation temperature that was $10^{\circ} \mathrm{C}$ higher than the non-permissive temperature of PlyC, however after screening 3,000 mutants, we were unable to identify any advantageous mutations. Keeping in mind directed evolution methodologies consist of sequentially identifying beneficial amino acid substitutions that have additive effects, it was determined that a less stringent temperature should be used during the screening process even if it increased the chance of generating false-positives. As such, we used an incubation temperature that was only a few degrees above the nonpermissive temperature and rescreened selected candidates to rule out the false-positives.

We decided to utilize an incubation temperature that was not too temperate $\left(\leq 1^{\circ} \mathrm{C}\right.$ higher than lowest non-permissive temperature $)$ and conversely not too harsh $\left(\geq 5^{\circ} \mathrm{C}\right.$ higher than lowest non-permissive temperature). The ideal temperature we decided to use in this particular assay was a moderate $2{ }^{\circ} \mathrm{C}$ higher than the experimentally determined non-permissive temperature. Choosing a temperature that is too mild will result in a much higher false positive identification rate than the $\sim 20 \%$ we observed (Table I) when using a moderate incubation temperature. Furthermore, using an incubation temperature that is too harsh could ultimately result in the inability to identify mutants with significantly enhanced thermal behavior. For example, using an incubation temperature of $\geq 5^{\circ} \mathrm{C}$ would have resulted in the inability to identify the promising $29 \mathrm{C} 3$ mutant as well as the majority of the other candidates selected during the first round of screening.

In summation, we provide a protocol for engineering bacteriolytic enzymes to acquire enhanced kinetic stability. Moreover, this same assay, without the heating steps, can be used to screen for mutations that increase catalytic activity. Augmenting both catalytic activity and thermostability is an important developmental hurdle facing any therapeutic enzyme. While the details of this protocol are specific to the endolysin PlyC, the methodology can be adapted to any bacteriolytic enzyme with only a few alterations. We presented preliminary results from only the first round of mutagenesis that validates that functionality of the assay, resulting in the implementation and identification of mutations that generate an increase in molecular thermostability of significant magnitude.

\section{Disclosures}

No conflicts of interest declared.

\section{Acknowledgements}

The authors wish to thank Emilija Renke and Janet Yu for technical assistance. DCN is supported by grants from the United States Department of Defense (DR080205, DM102823, OR09055, and OR090059). RDH is supported by a grant from the Maryland Agriculture Experiment Station and an NIH Training Grant in Cell and Molecular Biology.

\section{References}

1. Jackel, C., Kast, P., \& Hilvert, D. Protein design by directed evolution. Annual review of biophysics. 37, 153-173, doi:10.1146/ annurev.biophys.37.032807.125832 (2008). 
2. Liu, L., Li, Y., Liotta, D., \& Lutz, S. Directed evolution of an orthogonal nucleoside analog kinase via fluorescence-activated cell sorting. Nucleic acids research. 37, 4472-4481, doi:10.1093/nar/gkp400 (2009).

3. Fischetti, V.A. Bacteriophage lysins as effective antibacterials. Curr. Opin. Microbiol. 11, 393-400 (2008).

4. Fischetti, V.A., Nelson, D., \& Schuch, R. Reinventing phage therapy: are the parts greater than the sum? Nat. Biotechnol. 24, 1508-1511 (2006).

5. Loessner, M.J. Bacteriophage endolysins--current state of research and applications. Curr. Opin. Microbiol. 8, 480-487 (2005).

6. Nelson, D., Schuch, R., Chahales, P., Zhu, S., \& Fischetti, V.A. PlyC: A multimeric bacteriophage lysin. Proc. Natl. Acad. Sci. U.S.A. 103, 10765-10770 (2006).

7. Nelson, D., Loomis, L., \& Fischetti, V.A. Prevention and elimination of upper respiratory colonization of mice by group A streptococci using a bacteriophage lytic enzyme. Proc. Natl. Acad. Sci. U.S.A. 98, 4107-4112 (2001).

8. Filatova, L.Y., Becker, S.C., Donovan, D.M., Gladilin, A.K., \& Klyachko, N.L. LysK, the enzyme lysing Staphylococcus aureus cells: specific kinetic features and approaches towards stabilization. Biochimie. 92, 507-513, [pii] S0300-9084(10)00044-1 doi:10.1016/j.biochi.2010.01.026 (2010).

9. Varea, J., et al. Structural and thermodynamic characterization of Pal, a phage natural chimeric lysin active against pneumococci. J. Biol. Chem. 279, 43697-43707, [pii] M407067200 doi:10.1074/jbc.M407067200 (2004).

10. Sanz, J.M., Garcia, J.L., Laynez, J., Usobiaga, P., \& Menendez, M. Thermal stability and cooperative domains of CPL1 lysozyme and its NH2- and COOH-terminal modules. Dependence on choline binding. J. Biol. Chem. 268, 6125-6130 (1993).

11. Anderson, G. \& Scott, M. Determination of product shelf life and activation energy for five drugs of abuse. Clin. Chem. 37, 398-402 (1991).

12. Kim, G.J., Cheon, Y.H., \& Kim, H.S. Directed evolution of a novel N-carbamylase/D-hydantoinase fusion enzyme for functional expression with enhanced stability. Biotechnol. Bioeng. 68, 211-217 (2000).

13. Koutsioulis, D., et al. Directed evolution on the cold adapted properties of TAB5 alkaline phosphatase. Protein Eng. Des. Sel. 21, 319-327, doi:10.1093/protein/gzn009 (2008).

14. McCarthy, J.K., Uzelac, A., Davis, D.F., \& Eveleigh, D.E. Improved catalytic efficiency and active site modification of 1,4-beta-D-glucan glucohydrolase A from Thermotoga neapolitana by directed evolution. J. Biol. Chem. 279, 11495-11502, doi:10.1074/jbc.M305642200 (2004).

15. Ren, C., Chen, T., Zhang, J., Liang, L., \& Lin, Z. An evolved xylose transporter from Zymomonas mobilis enhances sugar transport in Escherichia coli. Microbial cell factories. 8, 66, doi:10.1186/1475-2859-8-66 (2009).

16. Tsuzuki, K., et al. Thermostable mutants of the photoprotein aequorin obtained by in vitro evolution. J. Biol. Chem. 280, 34324-34331, doi:10.1074/jbc.M505303200 (2005).

17. Eijsink, V.G., Gaseidnes, S., Borchert, T.V., \& van den Burg, B. Directed evolution of enzyme stability. Biomolecular engineering. 22, 21-30, doi:10.1016/j.bioeng.2004.12.003 (2005).

18. Kumar, S. \& Nussinov, R. How do thermophilic proteins deal with heat? Cell. Mol. Life Sci. 58, 1216-1233 (2001).

19. Hibbert, E.G. \& Dalby, P.A. Directed evolution strategies for improved enzymatic performance. Microb. Cell Fact. 4, 29, [pii] 1475-2859-4-29 doi:10.1186/1475-2859-4-29 (2005).

20. Rasila, T.S., Pajunen, M.I., \& Savilahti, H. Critical evaluation of random mutagenesis by error-prone polymerase chain reaction protocols, Escherichia coli mutator strain, and hydroxylamine treatment. Anal. Biochem. 388, 71-80, [pii] S0003-2697(09)00095-5 doi:10.1016/ j.ab.2009.02.008 (2009).

21. Wong, T.S., Roccatano, D., Zacharias, M., \& Schwaneberg, U. A statistical analysis of random mutagenesis methods used for directed protein evolution. J. Mol. Biol. 355, 858-871, doi:10.1016/j.jmb.2005.10.082 (2006).

22. Arnold, F.H., Wintrode, P.L., Miyazaki, K., \& Gershenson, A. How enzymes adapt: lessons from directed evolution. Trends in biochemical sciences. 26, 100-106 (2001). 